\title{
Suporte Social e Individuação em Jovens de Diferentes Configurações Familiares
}

\author{
Ana Raquel Silva ${ }^{1}$ \\ Olga Melo \\ Catarina Pinheiro Mota \\ Departamento de Educação e Psicologia da Universidade de Trás-os-Montes e Alto Douro, \\ Vila Real, Portugal \\ Centro de Psicologia da Universidade do Porto, Porto, Portugal
}

\begin{abstract}
Resumo
O estabelecimento de laços com a família, amigos ou mesmo professores é crucial para o desenvolvimento dos adolescentes e jovens adultos. Uma individuação bem-sucedida na adolescência implica qualidade de relação. O presente estudo tem como objetivo analisar as diferenças na percepção do suporte social e processo de individuação em função da configuração familiar, bem como analisar o poder preditor da configuração familiar, do gênero, da idade e da percepção do suporte social dos jovens no processo de individuação. A amostra foi constituída por 827 jovens a frequentarem o ensino secundário e universitário, com idades entre os 13 e os 25 anos. A coleta de dados foi realizada no Norte de Portugal, mediante questionários de auto-relato: Social Support Appraisals (SSA) e Munich Individuation Test of Adolescence (MITA). Os resultados mostraram que jovens de famílias intactas apresentavam uma individuação mais bem-sucedida comparativamente com jovens de famílias divorciadas, e ainda evidenciaram o poder preditor da percepção do suporte da família no desenvolvimento da individuação. Neste sentido, verificou-se a importância da configuração e do suporte familiar para uma individuação bem-sucedida.
\end{abstract}

Palavras-chave: Suporte social, individuação, divórcio.

\section{Social Support and Young People Individuation in Different Family Configurations}

\begin{abstract}
The establishment of bonds with family, friends or teachers is crucial to the development of adolescents and young adults. A successful individuation in adolescence involves the quality of relationships. This study aims to analyze the differences in the perception of social support and individuation process according to the family setting and to analyze the predictive power of family structure, gender, age and perceived social support of young people in the process of individuation. The sample consisted of 827 young people to attend secondary school and university, aged between 13 and 25 years. The data collection was carried in northern Portugal, through self-report questionnaires: Social Support Appraisals
\end{abstract}

Endereço para correspondência: Universidade de Trás-os-Montes e Alto Douro, Departamento de Educação e Psicologia, Edifício do Complexo Pedagógico, Quinta dos Prados, Vila Real, Portugal 5001-801. E-mail: a.raquel.f.silva@hotmail.com,olga_soaresmelo@hotmail.com e catppmota@utad.pt

Esta investigação foi parcialmente suportada pela Fundação para a Ciência e Tecnologia - FCT através do projecto: PEst-C/PSI/UI0050/2011 e ainda pelos fundos FEDER através do programa COMPETE com a referência: FCOMP-01-0124-FEDER-022714. 
(SSA) and Munich Individuation Test of Adolescence (MITA). The results showed that young peole of intact families had a more successful individuation compared to young people from divorced families, and showed the predictive power of family support perception in the development of individuation. In this sense, this study showed the importance of setting and family support to a successful individuation.

Keywords: Social support, individuation, divorce.

\section{El Apoyo Social y la Individuación en Jóvenes de Distintas Configuraciones Familiares}

\section{Resumen}

El establecimiento de vínculos con la familia, amigos o profesores es crucial para el desarrollo de los adolescentes y adultos jóvenes. El éxito del proceso de individuación en la adolescencia implica calidad en las relaciones. Este estudio tiene como objetivo analizar las diferencias en la percepción de apoyo social y el proceso de individuación de acuerdo con el entorno familiar y analizar la capacidad de predicción de la estructura familiar, el género, la edad y el apoyo social percibido de los jóvenes en el proceso de individuación. La muestra consistió en 827 jóvenes que asisten a la escuela secundaria y la universidad, con edades comprendidas entre 13 y 25 años. La recolección de datos se realizó en el norte de Portugal, a través de cuestionarios de autoinforme: Social Support Appraisals (SSA) e Munich Individuation Test of Adolescence (MITA). Los resultados mostraron que los jóvenes de familias intactas tenían una mejor individuación en comparación con los jóvenes de familias divorciadas, y mostraron el poder predictivo de la percepción de apoyo familiar en el desarrollo de la individuación. En este sentido, este estudio demostró la importancia del entorno y del apoyo familiar en el éxito del proceso de individuación de los jóvenes.

Palabras clave: Apoyo social, individuación, divorcio.

A percepção do suporte social implica uma avaliação global que envolve as várias áreas sociais da vida do indivíduo. Dessa avaliação pode resultar a crença de que se é amado (Heller, Swindle, \& Dusenbury, 1986).

Coob (1976) define suporte social em três classes: a primeira refere-se à percepção que o sujeito tem de que é amado e que recebe a preocupação por parte dos outros, a segunda diz respeito à crença que o sujeito tem de que é apreciado e valorizado, e a terceira reporta ao sentimento de pertença a uma rede de comunicação e de obrigações mútuas.

Antunes e Fontaine (1995) referem que o desenvolvimento de uma rede social ocorre ao longo da vida de cada indivíduo, sendo que este influencia e é influenciado pelo contexto e o meio social. Contudo, mais importante do que o apoio social efetivo que cada indivíduo recebe é a percepção que realiza do mesmo (Antunes $\&$ Fontaine, 2005). As redes sociais e o apoio social são importantes fatores num período de desenvolvimento como a adolescência, onde ocorre um aumento das redes sociais, podendo o apoio e a influência dessas redes variar ao longo do tempo (Antunes \& Fontaine, 2005). O estudo de Hombrados-Mendieta, Gomez-Jacinto, Dominguez-Fuentes, Garcia-Leiva, e Castro-Travé (2012) com 447 adolescentes com idades entre os 12 e os 18 anos, analisou as diferenças existentes entre os vários sistemas de suporte social, distinguindo entre o suporte fornecido pela família e pela escola. Os autores constataram que na adolescência há uma tendência para a diminuição da percepção de suporte dos pais e aumento da percepção de suporte dos colegas de escola.

Para Furman (2001) as relações de amizade, apesar de não funcionarem como bases seguras de vinculação, funcionam como "figuras de afiliação", onde à semelhança da vinculação a afiliação é descrita como um sistema de comportamentos que proporciona ao indivíduo relações de suporte, proteção, cooperação, mutualidade e reciprocidade. No decorrer da construção da 
identidade surge um maior recurso ao suporte dos pares, onde se evidencia uma maior interação social que vai para além da família (Sérgio, 2006).

Assim, verifica-se que tanto a ligação com a família, como com os pares, e ainda com os professores, também eles vistos como importante fonte de suporte social (Antunes 2006), são revelantes na autovalorização do adolescente, bem como no desenvolvimento de processos de separação e autonomia mais ou menos saudáveis. Segundo Meeus, Iedema, Maassen e Engels (2005), a separação entre filhos e pais e a individuação são dois processos desenvolvimentais que ocorrem em paralelo durante a adolescência, não se tratando, portanto, a separação como uma pré-condição para a individuação. Apesar da origem do termo individuação ter surgido com Margret Mahler, em 1930 (Mahler, Pine, \& Bergman, 1975), foi Blos (1967) que clarificou o conceito de segunda individuação, que ocorre na adolescência. Sendo que é nesta fase que o adolescente ganha determinadas competências, nomeadamente, ao nível da sua autonomia, havendo uma separação mútua relativamente aos pais.

Dentro do conceito de individuação na adolescência, Blos (1967) refere a importância de dois fenômenos, nomeadamente, a perda da dependência emocional e afetividade familiar e a mudança das relações objetais que se direcionavam para os pais e agora estão mais voltadas para os pares. A individuação na adolescência caracteriza-se pelo esforço dos adolescentes em alcançar a autonomia e o ato dos pais concederem liberdade aos filhos, sendo para isso necessário abdicarem do controle para lhes proporcionar essa mesma autonomia, o que, por vezes, causa afastamento entre pais e filhos. Este fato pode dificultar o processo de individuação, pois o apoio dos pais para a aquisição de autonomia é fundamental (Walper, Kruse, Noack, \& Schwarz, 2009).

Todos os desejos inerentes ao processo de individuação (desejo pela autonomia, desejo de "ser adulto" e desejo de explorar) fazemse acompanhar de um sentimento de medo em enfrentar a novidade. Assim, torna-se importante realçar neste ponto a importância da qualidade da relação parental com os filhos para que este processo se desenvolva de modo a permitir ao jovem sentir-se suficientemente seguro para explorar o meio, tornando-se mais autônomo e poder construir a sua identidade (Fleming, 2005).

A presente investigação pretende ir além do período da adolescência e alcançar o início da vida adulta, que Arnett (2007) designa como a adultez emergente que ronda os 18 e os 25 anos cronológicos. Também nesta fase o processo de individuação se desenvolve, merecendo um olhar atento de autores como Arnett (2006) e Bynner (2005) que consideram que o mesmo difere em função de fatores contextuais que rodeiam os jovens.

Existem alguns fatores passíveis de interferir no desenvolvimento da autonomia nos adolescentes e jovens adultos, como as descontinuidades emocionais. O divórcio parental é uma das muitas situações que podem afetar o desenvolvimento que acompanha a adolescência e a fase inicial da vida adulta, realçando-se, o processo de individuação. Freeman e Newland (2002) apontam que em situação de divórcio existe uma tendência para os adolescentes desenvolverem uma prematura independência face à família de uma das figuras parentais, o que pode prejudicar de forma significativa o desenvolvimento da individuação. Storksen, Roysamb, Moum e Tambs (2005) referem que crianças cujos pais atravessam um divórcio têm um maior risco de vir a ter problemas de adaptação durante a infância, a adolescência e até mesmo na vida adulta. Segundo Amato (2000), os filhos de famílias que sofrem um divórcio podem desenvolver mais dificuldades no envolvimento social. Contudo, de acordo com Amato e Cheadle (2008) esta ideia não pode ser generalizável, dado que nem todas as crianças expostas a situação de divórcio ou conflitos parentais desenvolvem problemas. Logo, tal como apontam Souza e Ramires (2006), não é possível afirmar uma relação de causa-efeito entre o divórcio e consequências negativas. Pelo contrário, é importante compreender toda a multiplicidade e complexidade de fatores envolvidos no processo de desenvol- 
vimento, podendo assumir contornos mais ou menos adaptativos.

A separação parental é uma situação que pode envolver um número considerável de indutores de stress nos membros da família, principalmente nos primeiros dois anos, onde decorrem as primeiras transformações no que diz respeito às rotinas, residência e configuração familiar (Amato \& Anthony, 2014). Dadas as experiências inerentes a esta situação, como os conflitos interparentais e separações, Amato e Dorius (2010) sugerem a possibilidade de os filhos desenvolverem uma série de problemas no decorrer das suas vidas. Os mesmos autores apontam que filhos de pais divorciados tendem a apresentar mais dificuldades no campo emocional, comportamental, social, saúde e acadêmico. Outra das consequências do divórcio dos pais é o afastamento entre os filhos e umas das figuras parentais, geralmente o pai, contudo importa referir que esta tendência tem vindo a diminuir, sendo que a frequência de contacto entre pais divorciados, que não possuem a tutela dos menores, e filhos tem vindo a aumentar nos últimos anos (Amato \& Dorius, 2010). Uma separação entre as figuras parentais implica muitas vezes mudanças de estilos de vida, de local de residência e mudança de meio envolvente, que podem constituir fatores stressantes para os filhos (Amato, 2000). Ao longo do processo de divórcio é comum a procura de suporte junto do grupo de pares (Moura \& Matos, 2008), funcionando como um porto seguro para os adolescentes e jovens adultos, o que não substitui a relação de vínculo com os pais. $\mathrm{O}$ grupo de pares pode proporcionar aos jovens que atravessam um processo de divórcio parental apoio físico e social, permitindo-lhes deste modo um melhor desenvolvimento do processo de individuação (Fleming, 2005). Ainda assim, a qualidade da vinculação aos pais é relevante para o bem-estar dos filhos numa situação de divórcio (Ramires, 2004), o que evidencia a importância da vinculação em detrimento do tipo de configuração familiar para o desenvolvimento dos adolescentes e jovens. Hack e Ramires (2010) ressaltam também a importância da qualidade da relação entre pais e filhos e a manutenção da mesma durante e após o divórcio. Freeman e Newland (2002) acrescentam a importância de realizar uma gestão entre a manutenção da relação com os pais e o alcance da autonomia, própria da individuação.

Para além do tipo de configuração familiar, também o sexo e a idade dos adolescentes e jovens pode se associar à existência de diferenças no desenvolvimento das suas relações, na perceção do suporte social e autonomia. A literatura aponta maioritariamente, que são as meninas que apresentam maior percepção de suporte social comparativamente com os meninos. Um estudo de Ikiz e Cakar (2010) com uma amostra de 257 adolescentes mostra que são as meninas que apresentam maior percepção do suporte social dos amigos comparativamente com os rapazes. Outros estudos sobre gênero e suporte social (Pinheiro \& Ferreira, 2005; Ramalho, 2008) obtiveram resultados semelhantes, evidenciando que as meninas possuem maior suporte social da família e dos amigos comparativamente com o sexo masculino. Antunes e Fontaine (2005), numa análise das qualidades psicométricas da Escala de Percepção do Suporte Social com 1963 estudantes do $7^{\circ}$ ao $12^{\circ}$ ano, evidenciaram que as meninas e os jovens mais velhos apresentavam em geral níveis mais elevados de suporte social, comparativamente com jovens mais novos e do sexo masculino. O que vai ao encontro também do estudo de Ramalho (2008) com 200 indivíduos entre os 18 e os 25 anos, que, da mesma forma, constatou que são os jovens mais velhos que possuem maior perceção do suporte social. Beato (2008) no seu estudo com 200 elementos, com idades entre os 17 e os 21 anos, aponta para a tendência dos rapazes apresentarem uma menor expressão afetiva em várias áreas da vida afetiva, sendo mais retractivos e menos envolvidos afetivamente. Para o mesmo autor esta falta de expressividade poderá estar relacionada com as práticas educativas orientadas para o valor próprio e não para as relações com os outros (Beato, 2008), o que poderá traduzir processos de individuação mais bem-sucedidos pelas meninas.

Com o presente estudo pretende-se analisar as diferenças na percepção do suporte social e 
processo de individuação em função da configuração familiar, bem como analisar o poder preditor da configuração familiar, do gênero, da idade e da percepção do suporte social dos jovens no processo de individuação.

\section{Método}

\section{Participantes}

A presente amostra foi constituída por 827 adolescentes e jovens adultos portugueses, sendo $253(30,6 \%)$ do sexo masculino e $574(69,4 \%)$ do sexo feminino, com idades compreendidas entre os 13 e os 25 anos $(M=17,17 ; D P= \pm 3,28)$. No que se refere ao nível de escolaridade 296 $(35,8 \%)$ sujeitos frequentam o $3^{\circ}$ ciclo (ensino fundamental no $7^{\circ}, 8^{\circ}$ e $9^{\circ}$ ano, no Brasil), 213 $(25,8 \%)$ frequentam o ensino secundário (ensino médio, no Brasil) e 318 (38,5\%) frequentam o ensino superior $(M=10,70 ; D P= \pm 2,14)$. No que respeita à configuração familiar da amostra 574 $(69,4 \%)$ jovens pertencem a famílias intactas, cujos pais estavam casados ou em união de fato (união estável, no Brasil), 245 (29,6\%) jovens pertencem a famílias divorciadas, cujos pais estavam divorciados ou separados e $8(1 \%)$ jovens em que os pais são viúvos ou solteiros.

\section{Instrumentos}

Foi elaborado um questionário sociodemográfico com vista à recolha de informação referente a dados sociodemográficos, nomeadamente, idade, sexo, ano de escolaridade, escola ou universidade que frequenta e configuração do agregado familiar.

Para avaliar a variável suporte social foi utilizada uma adaptação da Escala "Social $\mathbf{S u}$ pport Appraisals" (SSA), de Vaux e colaboradores (1980), realizada por Antunes e Fontaine (1995). A presente escala avalia a percepção do apoio social dos adolescentes a quatro níveis, nomeadamente, o apoio emocional dos amigos com 7 itens, por exemplo, "os meus amigos respeitam-me", o apoio emocional da família com 8 itens, por exemplo, "a minha família estima-me muito", o apoio emocional geral com 8 itens, por exemplo, "as pessoas, de um modo geral, gostam de mim", e ainda, o apoio emocio- nal dos professores com 8 itens, por exemplo, "tenho professores que se preocupam bastante comigo", concluindo-se um total de 30 itens na versão portuguesa depois de alargada, onde foi acrescentada a sub-escala apoio emocional dos professores com 8 itens. Trata-se de uma escala que avalia em 4 pontos ("concordo totalmente", "concordo", "discordo", "discordo totalmente"), devendo-se ter em consideração que metade dos seus itens são positivos e outra metade negativos. Relativamente às suas propriedades psicométricas a escala apresenta um bom poder discriminativo e uma boa consistência interna global ( $\alpha=0,91$; Antunes \& Fontaine, 1995). Na presente amostra foram encontrados os seguintes valores de consistência interna, para a dimensão amigos 0,85 , para a dimensão família 0,88 , para a dimensão professor 0,82 e para a dimensão geral 0,64 , sendo o valor de consistência interna da escala completa de 0,89 . A análise fatorial confirmatória à escala SSA demonstra que esta apresenta um ajustamento adequado ${ }^{2}(44)=$ 321,02, $p=0,001$; $\mathrm{CFI}=0,94$, RMSEA $=0,09$; (S) $\mathrm{RMR}=0,05$.

Para medir o processo de individuação utilizou-se o Munich Individuation Test of Adolescence (MITA) de Walper, Schwarz, e Jurasic (1996), nomeadamente a versão de investigação de Matos e Hüsgen (2009) para a população portuguesa. A escala referida encontra-se dividida de igual forma para o adolescente avaliar a mãe e o pai e é constituída por 27 itens, agrupados em 6 dimensões: manutenção do laço, negação do desejo de ligação, individuação bem sucedida, ansiedade de fusão e medo de controle, medo da perda de amor e ambivalência. Os três primeiros dizem respeito ao relacionamento e os três últimos às inseguranças em relação aos pais (Kruse \& Walper, 2008). É de referir que na presente investigação serão utilizadas apenas três dimensões que dizem respeito ao relacionamento ("relatedness"): manutenção do laço com 3 itens, por exemplo, "gostaria de viver coisas em conjunto com os meus pais", negação do desejo de ligação com 5 itens, por exemplo, "fico inseguro(a) quando temos opiniões diferentes" e individuação bem-sucedida com 4 itens, por exemplo, "os meus pais continuam a ser impor- 
tantes para mim, apesar de eu seguir os meus próprios interesses". De acordo com Kruse e Walper (2008) as escalas mostram consistência interna adequada que varia de alfa de Cronbach $\alpha=0,65$ a $\alpha=0,84$. Na presente amostra foram encontrados os seguintes valores de consistência interna para as dimensões referentes à mãe: manutenção do laço 0,69 , negação do desejo de ligação 0,67 e individuação bem-sucedida 0,77 . No que respeita ao pai, obtivemos os seguintes valores de consistência interna: manutenção do laço 0,77 , negação do desejo de ligação 0,65 e individuação bem-sucedida 0,85 . A análise fatorial confirmatória à versão Mãe da escala MITA demonstra que esta apresenta um ajustamento adequado ${ }^{2}(17)=35,73, p=0,005$; $\mathrm{CFI}=0,98 ; \mathrm{RMSEA}=0,03 ;(\mathrm{S}) \mathrm{RMR}=0,04$. No que respeita à versão Pai verifica-se que também apresenta um ajustamento adequado ${ }^{2}(17)=$ 98,65, $p=0,001 ; \mathrm{CFI}=0,97$; RMSEA $=0,05 ;(\mathrm{S})$ $\mathrm{RMR}=0,08$.

\section{Procedimentos}

Coleta de Dados. A presente investigação de natureza transversal realizou uma coleta de forma aleatória no Norte de Portugal em instituições como Escolas Secundárias e Escolas Profissionais em turmas do $7^{\circ}$ ao $12^{\circ}$ ano, e ainda na Universidade de Trás-os-Montes e Alto Douro e em população do Norte de Portugal, tratando-se de uma amostra de conveniência. A coleta de dados, através da administração do protocolo, foi realizada, após a devida autorização e consentimento informado dos representantes das instituições e dos participantes. Durante a coleta de dados a equipe de investigação procedeu ao fornecimento de uma instrução padrão, que esclareceu os objetivos e a finalidade do estudo. Todas as questões éticas foram garantidas, como a confidencialidade, anonimato e voluntariedade da participação.

Análise de Dados. Os dados foram analisados com recurso ao programa estatístico $\mathrm{Sa}$ tistical Package for the Social Sciences, versão 22. Procedeu-se à limpeza dos dados (missings e outliers) e ainda à análise das propriedades psicométricas, como a confirmação da normalidade da amostra, bem como consistência interna e análises fatoriais confirmatórias dos instrumentos. Para a obtenção dos resultados procedeu-se a análises estatísticas recorrendo às análises de variância entre grupos através do test- $t$ e análises de regressão múltipla hierárquica.

\section{Resultados}

\section{Percepção do Suporte Social e do Processo de Individuação em Função da Configuração Familiar}

Ao comparar as dimensões da percepção do suporte social em função da configuração familiar, foi possível inferir que não existem diferenças significativas entre jovens de famílias intactas e divorciadas nas dimensões amigos, professor e geral, enquanto na dimensão família ocorrem diferenças significativas entre jovens de diferentes configurações familiares, sendo que os jovens pertencentes a famílias intactas apresentam níveis superiores de percepção do suporte familiar comparativamente com os jovens de famílias divorciadas (Tabela 1). No que concerne à presença de diferenças significativas ao nível do processo de individuação em função da configuração familiar (jovens de famílias intactas e divorciadas) realizaram-se análises diferenciais para mãe e pai separadamente.

Relativamente à análise do processo de individuação à mãe, na dimensão manutenção do laço à mãe verifica-se que existem diferenças significativas entre os jovens de famílias intactas e divorciadas, denotando-se que os jovens de famílias intactas apresentam maior manutenção do laço com a mãe comparativamente aos jovens de famílias divorciadas. $\mathrm{Na}$ dimensão negação do desejo de ligação à mãe denota-se a existência de diferenças, sendo que os jovens que pertencem a famílias divorciadas apresentam maior negação do desejo de ligação em relação à mãe comparativamente com os jovens de famílias intactas. Analisando a individuação bem-sucedida à mãe evidenciam-se diferenças significativas, sendo que maiores níveis de individuação bem-sucedida à mãe pertencem aos jovens que têm uma estrutura familiar intacta comparando com os jovens de famílias divorciadas (Tabela 1). 
Tabela 1

Diferenciação da Percepção do Suporte Social e Processo de Individuação em Função da Configuração Familiar

\begin{tabular}{|c|c|c|c|c|c|}
\hline \multirow{3}{*}{ Dimensões } & 1-Família Intacta & 2-Família Divorciada & \multirow{3}{*}{$95 \% \mathrm{IC}$} & \multirow{3}{*}{$t$} & \multirow{3}{*}{$p$} \\
\hline & $(n=574)$ & $(n=245)$ & & & \\
\hline & $M \pm D P$ & $M \pm D P$ & & & \\
\hline \multicolumn{6}{|l|}{ SSA } \\
\hline Amigos & $4,94 \pm 0,75$ & $4,92 \pm 0,79$ & {$[-0,09 ; 0,14]$} & $t(81)=0,36$ & 0,716 \\
\hline Família & $5,23 \pm 0,73$ & $4,89 \pm 0,97$ & {$[0,23 ; 0,47]$} & $t(366)=5,06$ & 0,001 \\
\hline Professor & $4,00 \pm 0,86$ & $3,95 \pm 0,98$ & {$[-0,09 ; 0,19]$} & $t(414)=0,74$ & 0,459 \\
\hline Geral & $4,37 \pm 0,58$ & $4,30 \pm 0,66$ & {$[-0,03 ; 0,16]$} & $t(410)=1,35$ & 0,179 \\
\hline \multicolumn{6}{|l|}{ MITA MÃE } \\
\hline Manutenção do Laço & $3,33 \pm 0,56$ & $3,14 \pm 0,78$ & {$[0,09 ; 0,30]$} & $t(356)=3,54$ & 0,001 \\
\hline $\begin{array}{l}\text { Negação do Desejo } \\
\text { de Ligação }\end{array}$ & $1,83 \pm 0,56$ & $1,98 \pm 0,64$ & {$[-0,24 ;-0,05]$} & $t(415)=-3,07$ & 0,002 \\
\hline $\begin{array}{l}\text { Individuação } \\
\text { bem sucedida }\end{array}$ & $3,54 \pm 0,46$ & $3,35 \pm 0,72$ & {$[0,08 ; 0,28]$} & $t(332)=3,64$ & 0,002 \\
\hline \multicolumn{6}{|l|}{ MITA PAI } \\
\hline Manutenção do Laço & $3,29 \pm 0,61$ & $2,78 \pm 0,96$ & {$[0,38 ; 0,64]$} & $t(330)=7,67$ & 0,001 \\
\hline $\begin{array}{l}\text { Negação do Desejo } \\
\text { de Ligação }\end{array}$ & $1,88 \pm 0,58$ & $2,18 \pm 0,67$ & {$[-0,39 ;-0,20]$} & $t(404)=-6,06$ & 0,001 \\
\hline $\begin{array}{l}\text { Individuação } \\
\text { bem sucedida }\end{array}$ & $3,45 \pm 5,29$ & $2,92 \pm 0,97$ & {$[0,39 ; 0,66]$} & $t(308)=8,06$ & 0,001 \\
\hline
\end{tabular}

Nota. SSA- escala da percepção do suporte social; MITA- Munich Individuation Test of Adolescence.

No que respeita ao processo de individuação direcionado ao pai foi também possível verificar diferenças significativas. Na manutenção do laço ao pai verifica-se que os dois grupos de jovens apresentam diferenças significativas, denotando-se que os níveis mais altos pertencem aos jovens de famílias intactas comparativamente com os jovens de famílias divorciadas. Analisando a negação do desejo de ligação ao pai denota-se que do mesmo modo existem diferenças significativas entre grupos, sendo que são os jovens de famílias divorciadas que apresentam níveis superiores aos jovens de famílias intactas. Finalmente, analisando a individuação bem-sucedida em relação ao pai, verifica-se que também existem diferenças significativas nas médias entre grupos, verificando-se que são os jovens de famílias intactas que apresentam níveis superiores de individuação bem-sucedida ao pai comparando com os jovens de famílias divorciadas.
Predição do Processo de Individuação: Papel do Sexo, Configuração Familiar, Idade e Percepção do Suporte Social

Para analisar o poder preditor das variáveis sexo, configuração familiar, idade e da percepção do suporte social no processo de individuação recorreu-se à análise de regressão múltipla hierárquica. No que respeita às variáveis sexo, configuração familiar e idade foram criadas variáveis dummys.

Predição da Manutenção do Laço à Mãe e ao Pai. Analisando os resultados verifica-se que o modelo da dimensão manutenção do laço à mãe pode ser predito significativamente por algumas das variáveis independentes introduzidas. Neste sentido o sexo (bloco 1) tem um contributo significativo $F(1,821)=4,39 ; p=0,037$, explicando $0,5 \%$ da variância total $\left(R^{2}=0,005\right)$, contribuindo individualmente com $0,5 \%$ da variância para o modelo $\left(R^{2}\right.$ change $\left.=0,005\right)$. No 
bloco 2, surge a configuração familiar que também contribui significativamente para a variância do modelo $F(2,820)=9,66 ; p=0,001$ e explica $2,3 \%$ da variância total $\left(R^{2}=0,023\right)$, apresentando um contributo individual de $1,8 \%$ $\left(R^{2}\right.$ change $\left.=0,018\right)$. Também a percepção do suporte social (bloco 4) contribui significativamente para o modelo $F(7,815)=14,11 ; p=0,001$ e explica $10,8 \%$ da variância total $\left(R^{2}=0,108\right)$, apresentando um contributo individual de $8,4 \%$ ( $R^{2}$ change $\left.=0,084\right)$.
Realizando uma análise mais pormenorizada de cada variável independente verifica-se que o modelo da variável manutenção do laço à mãe recebe uma contribuição significativa de três variáveis que explicam $10,8 \%$ do modelo. As variáveis são as apresentadas por ordem de importância: percepção do suporte da família $(\beta=0,297 ; p=0,001)$; famílias intactas $(\beta=-$ $0,075 ; p=0,029)$; e percepção do suporte do professor $(\beta=0,073 ; p=0,046$; Tabela 2$)$.

\section{Tabela 2}

Regressões Múltiplas Hierárquicas para Processo de Individuação

\begin{tabular}{|c|c|c|c|c|c|c|c|}
\hline Manutenção do laço à mãe & $R^{2}$ & $R^{2}$ Change & $\mathrm{B}$ & $S E$ & $\beta$ & $t$ & $p$ \\
\hline Bloco 1- Sexo & 0,005 & 0,005 & & & & & \\
\hline Bloco 2 - Configuração f. & 0,023 & 0,018 & $-0,104$ & 0,048 & $-0,075$ & 2,187 & 0,029 \\
\hline Bloco 3 - Idade & 0,024 & 0,001 & & & & & \\
\hline Bloco 4 - SSA & 0,108 & 0,084 & & & & & \\
\hline \multicolumn{8}{|l|}{ Amigos } \\
\hline Família & & & 0,233 & 0,032 & 0,297 & 7,189 & 0,001 \\
\hline Professor & & & 0,053 & 0,026 & 0,073 & 1,999 & 0,046 \\
\hline \multicolumn{8}{|l|}{ Geral } \\
\hline Manutenção do laço ao pai & $R^{2}$ & $R^{2}$ Change & $\mathrm{B}$ & $S E$ & $\beta$ & $t$ & $p$ \\
\hline Bloco 1 - Sexo & 0,001 & 0,001 & & & & & \\
\hline Bloco 2 - Configuração f. & 0,086 & 0,085 & $-0,417$ & 0,056 & $-0,251$ & 7,477 & 0,001 \\
\hline Bloco 3 - Idade & 0,086 & 0,000 & & & & & \\
\hline Bloco 4 - SSA & 0,132 & 0,046 & & & & & \\
\hline \multicolumn{8}{|l|}{ Amigos } \\
\hline Família & & & 0,222 & 0,038 & 0,237 & 5,819 & 0,001 \\
\hline \multicolumn{8}{|l|}{ Professor } \\
\hline \multicolumn{8}{|l|}{ Geral } \\
\hline Negação do desejo de ligação à mãe & $R^{2}$ & $R^{2}$ Change & $\mathrm{B}$ & $S E$ & $\beta$ & $t$ & $p$ \\
\hline Bloco $1-$ Sexo & 0,068 & 0,068 & 0,251 & 0,042 & 0,196 & 6,020 & 0,001 \\
\hline Bloco 2 - Configuração f. & 0,077 & 0,074 & & & & & \\
\hline Bloco 3 - Idade & 0,082 & 0,005 & $-0,084$ & 0,040 & $-0,068$ & 2,066 & 0,039 \\
\hline Bloco 4 - SSA & 0,179 & 0,097 & & & & & \\
\hline \multicolumn{8}{|l|}{ Amigos } \\
\hline Família & & & $-0,251$ & 0,028 & $-0,349$ & 8,809 & 0,001 \\
\hline \multicolumn{8}{|l|}{ Professor } \\
\hline Geral & & & 0,137 & 0,043 & 0,140 & 3,178 & 0,002 \\
\hline
\end{tabular}




\begin{tabular}{|c|c|c|c|c|c|c|c|}
\hline Negação do desejo de ligação ao pai & $R^{2}$ & $R^{2}$ Change & B & $S E$ & $\beta$ & $t$ & $p$ \\
\hline Bloco 1 - Sexo & 0,048 & 0,048 & 0,213 & 0,044 & 0,158 & 4,826 & 0,001 \\
\hline Bloco 2 - Configuração f. & 0,093 & 0,045 & 0,209 & 0,044 & 0,155 & 4,723 & 0,001 \\
\hline Bloco 3 - Idade & 0,094 & 0,002 & & & & & \\
\hline Bloco $4-$ SSA & 0,169 & 0,074 & & & & & \\
\hline \multicolumn{8}{|l|}{ Amigos } \\
\hline Família & & & $-0,235$ & 0,030 & $-0,310$ & 7,779 & 0,001 \\
\hline \multicolumn{8}{|l|}{ Professor } \\
\hline Geral & & & 0,125 & 0,046 & 0,122 & 2,747 & 0,006 \\
\hline Individuação bem-sucedida à mãe & $R^{2}$ & $R^{2}$ Change & B & $S E$ & $\beta$ & $t$ & $p$ \\
\hline Bloco $1-$ Sexo & 0,011 & 0,011 & & & & & \\
\hline Bloco 2 - Configuração f. & 0,031 & 0,020 & $-0,085$ & 0,040 & $-0,070$ & 2,145 & 0,032 \\
\hline Bloco 3 - Idade & 0,034 & 0,004 & & & & & \\
\hline Bloco $4-$ SSA & 0,180 & 0,145 & & & & & \\
\hline \multicolumn{8}{|l|}{ Amigos } \\
\hline Família & & & 0,232 & 0,027 & 0,340 & 8,600 & 0,001 \\
\hline \multicolumn{8}{|l|}{ Professor } \\
\hline \multicolumn{8}{|l|}{ Geral } \\
\hline Individuação bem-sucedida ao pai & $R^{2}$ & $R^{2}$ Change & B & $S E$ & $\beta$ & $t$ & $p$ \\
\hline Bloco $1-$ Sexo & 0,000 & 0,000 & & & & & \\
\hline Bloco 2 - Configuração f. & 0,109 & 0,108 & $-0,441$ & 0,051 & $-0,280$ & 8,575 & 0,001 \\
\hline Bloco 3 - Idade & 0,109 & 0,000 & & & & & \\
\hline Bloco $4-$ SSA & 0,183 & 0,075 & & & & & \\
\hline \multicolumn{8}{|l|}{ Amigos } \\
\hline Família & & & 0,243 & 0,035 & 0,274 & 6,935 & 0,001 \\
\hline \multicolumn{8}{|l|}{ Professor } \\
\hline Geral & & & & & & & \\
\hline
\end{tabular}

Nota. B, SE para um nível de significância de $p<0,05$; Bloco 1- Sexo; Bloco 2- Configuração Familiar; Bloco 3 - Idade; Bloco 4- Dimensões da percepção do suporte social (SSA).

Relativamente à dimensão manutenção do laço ao pai verifica-se que a configuração familiar (bloco 2) contribui significativamente para a variância do modelo $F(2,820)=38,38 ; p=0,001$, explicando $8,6 \%$ da variância total $\left(R^{2}=0,086\right)$, e apresentando um contributo individual de $8,5 \%\left(R^{2}\right.$ change $\left.=0,085\right)$. Também a percepção do suporte social contribui significativamente para o modelo $F(7,815)=17,66 ; p=0,001 \mathrm{e}$ explica $13,2 \%$ da variância total $\left(R^{2}=0,132\right)$, apresentando um contributo individual de 4,6\% $\left(R^{2}\right.$ change $\left.=0,046\right)$. Através de uma análise mais aprofundadamente de cada variável independente verifica-se que o modelo da variável manutenção do laço ao pai recebe uma contribuição significativa de duas variáveis que explicam $13,2 \%$ da variância do modelo. Assim, a configuração familiar - famílias casadas contribui de 
forma significativa para a explicação do modelo ( $\beta=-0,251 ; p=0,001)$, do mesmo modo também a percepção do suporte da família tem também um contributo significativo para a explicação do modelo ( $\beta=0,237 ; p=0,001$; ver Tabela 2$)$.

Predição da Negação do Desejo de Ligação à Mãe e ao Pai. Relativamente à dimensão negação do desejo de ligação à mãe introduziu-se no bloco 1 o sexo, este tem um contributo significativo $F(1,821)=59,93 ; p=0,001$ explicando $6,8 \%$ da variância total $\left(R^{2}=0,068\right)$, contribuindo individualmente com $6,8 \%$ da variância para o modelo ( $R^{2}$ change $\left.=0,068\right)$. No bloco 2 , a configuração familiar também contribui significativamente para a variância do modelo $F(2$, $820)=33,99 ; p=0,001$ e explica $7,7 \%$ da variância total $\left(R^{2}=0,077\right)$, apresentando um contributo individual de $0,9 \%\left(R^{2}\right.$ change $\left.=0,009\right)$. Relativamente ao bloco 3, este também contribui significativamente para o modelo $F(3,819)=24,26$; $p=0,001$, explicando $8,2 \%$ da variância total $\left(R^{2}=0,082\right)$, revelando um contributo individual de $0,5 \%\left(R^{2}\right.$ change $\left.=0,005\right)$. No bloco 4 , consta a percepção do suporte social que contribui significativamente para o modelo $F(7,815)=25,39$; $p=0,001$ e explica $17,2 \%$ da variância total $\left(R^{2}=0,172\right)$, apresentando um contributo individual de $9,7 \%\left(R^{2}\right.$ change $\left.=0,097\right)$. Através de uma análise mais aprofundadamente de cada variável independente verifica-se que o modelo da variável negação do desejo de ligação à mãe recebe uma contribuição significativa de quatro variáveis que explicam $17,2 \%$ da variância do modelo. As variáveis contribuintes são as apresentadas por ordem de importância: percepção do suporte da família ( $\beta=-0,349 ; p=0,001)$; sexo masculino ( $\beta=0,196 ; p=0,001)$; percepção do suporte social geral $(\beta=0,140 ; p=0,002)$; e, idade dos 13 aos 18 anos $(\beta=-0,068 ; p=0,039$; Tabela 2$)$.

Relativamente ao modelo negação do desejo de ligação ao pai os resultados evidenciam que o sexo (bloco 1), apresenta um contributo significativo $F(1,821)=41,44 ; p=0,001$ explicando $4,8 \%$ da variância total $\left(R^{2}=0,048\right)$, contribuindo individualmente com $4,8 \%$ da variância para o modelo $\left(R^{2}\right.$ change $\left.=0,048\right)$. Também a configuração familiar (bloco 2 ) contribui significativamente para a variância do modelo $F(2$,
$820)=41,83 ; p=0,001$ e explica $9,3 \%$ da variância total $\left(R^{2}=0,093\right)$, apresentando um contributo individual de $4,5 \%$ ( $R^{2}$ change $\left.=0,045\right)$. No bloco 4 , consta a percepção do suporte social que da mesma forma contribui significativamente para o modelo $F(7,815)=23,61 ; p=0,001$ e explica $16,9 \%$ da variância total $\left(R^{2}=0,169\right)$, apresentando um contributo individual de 7,4\% $\left(R^{2}\right.$ change $\left.=0,074\right)$. Analisando cada uma das dimensões particularmente é possível verificar que o modelo da variável negação do desejo de ligação ao pai recebe uma contribuição significativa de quatro variáveis que explicam $16,9 \%$ da variância do modelo. As variáveis contribuintes são as apresentadas por ordem de importância: percepção do suporte da família $(\beta=-0,310$; $p=0,001)$; sexo masculino $(\beta=0,158 ; p=0,001)$; famílias divorciadas $(\beta=0,155 ; p=0,001) ; \mathrm{e}$ percepção do suporte social geral $(\beta=0,122$; $p=0,006$; Tabela 2).

Predição da Individuação Bem-Sucedida à Mãe e ao Pai. Analisando a dimensão individuação bem-sucedida à mãe verifica-se que em relação ao primeiro bloco sexo, existe um contributo significativo $F(1,821)=8,95 ; p=0,003$, explicando $1,1 \%$ da variância total $\left(R^{2}=0,011\right)$, com uma contribuição individual de $1,1 \%$ da variância para o modelo $\left(R^{2}\right.$ change $\left.=0,011\right)$. No bloco 2 , configuração familiar apresenta igualmente uma contribuição significativa para a variância do modelo $F(2,820)=13,07 ; p=0,001$, explicando $3,1 \%$ da variância total $\left(R^{2}=0,031\right)$, com um contributo individual de $2 \%$ ( $R^{2}$ chan$g e=0,020)$. Da mesma forma, a percepção do suporte social contribui significativamente para o modelo $F(7,815)=25,48 ; p=0,001$ e explica $18 \%$ da variância total $\left(R^{2}=0,180\right)$, apresentando um contributo individual de $14,5 \%\left(R^{2}\right.$ chan$g e=0,145)$. Analisando o contributo individual de cada variável independente verifica-se que duas variáveis explicam $14,5 \%$ da variância total do modelo da individuação bem-sucedida, sendo elas a percepção do suporte da família $(\beta=0,340 ; p=0,001)$ e a configuração familiar famílias casadas $(\beta=-0,070 ; p=0,032)$.

Ao analisar os resultados relativos à dimensão individuação bem-sucedida ao pai verifica-se que a configuração familiar (blo- 
co 2) evidencia uma contribuição significativa para a variância do modelo $F(2,820)=49,90$; $p=0,001$, explicando $10,9 \%$ da variância total $\left(R^{2}=0,109\right)$, com um contributo individual de $10,8 \%$ ( $R^{2}$ change $\left.=0,108\right)$. Também a percepção do suporte social (bloco 4 ) contribui significativamente para o modelo $F(7,815)=26,09$; $p=0,001$ e explica $18,3 \%$ da variância total $\left(R^{2}=0,183\right)$, apresentando um contributo individual de $7,5 \%$ ( $R^{2}$ change $\left.=0,075\right)$. Ao observar o contributo individual de cada variável independente verifica-se que apenas duas explicam $18,3 \%$ da variância total do modelo da individuação bem-sucedida ao pai, sendo elas a configuração familiar - famílias casadas $(\beta=-$ $0,280 ; p=0,001)$ e a percepção do suporte da família $(\beta=0,274 ; p=0,001$; Tabela 2$)$.

\section{Discussão}

O presente estudo teve como objetivo analisar as diferenças na percepção do suporte social e processo de individuação em função da configuração familiar, bem como analisar o poder preditor da configuração familiar, do gênero, da idade e da percepção do suporte social dos jovens no processo de individuação.

A percepção de suporte social é uma importante componente na vida dos adolescentes e jovens. As análises realizadas no presente estudo mostraram que são os jovens de famílias intactas que possuem melhor percepção do suporte da família, comparando com os jovens de famílias que passaram por separação ou divórcio. Talvez o fato dos divórcios e separações estarem associados a altos níveis de conflitos, na fase que antecede o divórcio, interfira na estabilidade relacional familiar, o que pode diminuir as trocas afetivas e a consequente percepção das mesmas. Os divórcios estão também muitas vezes associados a separações e quebras de vínculos dos jovens para com alguns elementos da família, como pai, mãe, irmãos e avós, o que pode causar uma diminuição do apoio familiar e da percepção do mesmo. Em última instância o divórcio parental pode implicar ruturas no holding familiar, ou seja, na estrutura de suporte emocional familiar, este fato pode gerar sentimentos de perda nos jovens que atravessam esta mudança na configuração familiar (Hack \& Ramires, 2010). Um estudo qualitativo de Brito (2007) com jovens entre os 21 e os 29 anos, filhos de pais divorciados, ao analisar as percepções dos filhos em relação às mudanças que ocorrem em consequência do divórcio parental, mostrou que a separação não se restringe às figuras parentais, mas alarga-se ao relacionamento entre pais e filhos. O mesmo estudo mostrou ainda que os jovens que mantiveram um estreito relacionamento com as duas figuras parentais apresentaram menor desgaste emocional com o divórcio parental. No entanto, o divórcio também pode ser um fator positivo para os filhos, uma vez que jovens de famílias divorciadas podem apresentar níveis mais satisfatórios de bem-estar, comparativamente com jovens de famílias casadas onde ocorrem conflitos (Amato \& Sobolewski, 2001). Brown (2009) acrescenta que a maior parte dos filhos, de pais divorciados, não sofreram efeitos negativos, a longo prazo, ao nível do ajustamento psicológico e social. Para aqueles que apresentam efeitos negativos, o autor explica que poderá estar relacionado com a experiência de níveis elevados de conflito interparental, perda de recursos econômicos, perda de suporte social e escasso contacto com um ou ambos os pais (Brown, 2009).

No que concerne ao processo de individuação à mãe e ao pai, foi possível observar que existem diferenças significativas em função da configuração familiar, sendo que os jovens que pertencem a família intactas possuem maiores níveis de manutenção do laço à mãe e ao pai e de individuação bem-sucedida à mãe e ao pai. Por outro lado, são os jovens que pertencem a famílias separadas ou divorciadas que apresentam níveis superiores de negação do desejo de ligação à mãe e ao pai, o que pode estar relacionado com o fato de uma das figuras parentais ter que abandonar o lar. O medo da perda também poderá explicar estes resultados, no sentido em que o medo de saírem magoados da situação de divórcio parental poderá levar os adolescentes e jovens a retraírem-se mais no processo de ligação aos pais. Os resultados obtidos vão ao encontro do estudo de Kruse e Walper (2008), rea- 
lizado com 649 participantes, com idade entre os 10 e os 20 anos, provenientes de diferentes contextos familiares. O referido estudo demonstrou que os adolescentes e jovens quando expostos a situações de conflitos parentais, muito comuns em situações de processo de divórcio, apresentam dificuldades no processo de individuação, o que pode estar relacionado com a diminuição da disponibilidade parental. Também o estudo de Parmiani, Iafrate, e Giuliani (2012) com 264 jovens italianos provenientes de famílias intactas e separadas, verificou que os jovens provenientes de famílias separadas revelavam dificuldades no processo de individuação.

No que respeita às análises de predição, os resultados evidenciam que a configuração familiar e a percepção do suporte social têm um contributo significativo para a manutenção do laço à mãe e ao pai. Numa análise mais detalhada foi possível verificar que a percepção do suporte da família é uma das dimensões que contribui significativamente para a manutenção do laço ao pai e à mãe, sendo que níveis elevados da percepção do suporte familiar predizem níveis elevados de manutenção do laço à mãe e ao pai. Os jovens ao perceberem mais disponibilidade afetiva por parte da família procuram apoio junto da mesma, havendo assim trocas recíprocas de afeto que ajudam à manutenção dos laços afetivos. Segundo Noom, Dekovic e Meeus (1999) a relação entre pais e filhos e a percepção de suporte afetivo por parte destes facilita o desenvolvimento da formação da identidade. Assim, os jovens apoiados e seguros sentem-se mais protegidos e capazes no que concerne ao desenvolvimento da sua autonomia. Segundo Arnett (2006) e Bynner (2005) o processo de separação e individuação dos jovens difere em função vários fatores socioeconômicos e contextuais. Bynner (2005) refere que existem fatores moderadores que podem gerar diferenças no processo de individuação, sendo que um desses fatores é a qualidade da relação com as figuras parentais.

Outra das dimensões que contribui de forma significativa para a manutenção do laço ao pai $\mathrm{e}$ à mãe é a configuração familiar, onde jovens de famílias intactas predizem níveis mais elevados de manutenção do laço ao pai e à mãe. Segun- do Kruse e Walper (2008) diferentes dinâmicas familiares promovem diferentes tipos de individuação, pelo que uma dinâmica familiar pautada por estabilidade e segurança poderá gerar uma maior proximidade quer física quer emocional entre pais e filhos, promovendo, mais facilmente, um ambiente de segurança para procura de apoio junto das figuras parentais. No entanto, possíveis alterações no comportamento ou dificuldades relacionais não têm que ser justificados a partir do divórcio parental por si só, sendo que as consequências de um divórcio dependem da atitude do casal, da presença ou não de conflitos e da forma como os filhos percebem e elaboram a situação (Mota \& Matos, 2011). Um estudo de Leme, Del Prette e Coimbra (2015) com 454 adolescentes de diferentes configurações familiares, mostra que o tipo de configuração familiar não é um fator preditor de bem-estar psicológico para os adolescentes, apresentando o suporte social (amigos e família) como uma possível explicação das variações do bem-estar psicológico dos adolescentes. Para Amato e Afifi (2006), quando a relação interparental é pautada por conflitos, o fato de não ocorrer um divórcio, não protege mais os seus filhos de possíveis efeitos negativos, dado que os conflitos, por si só, causam danos no bem-estar dos filhos.

Também a percepção do apoio dos professores contribui de forma significativa para o modelo da manutenção do laço à mãe, sendo que níveis elevados de percepção do suporte dos professores predizem níveis elevados de manutenção do laço à mãe. Ao perceberem apoio por parte do professor, o adolescente vai procurar investir mais nessa relação que pode trazer benefícios para ele. Assim, uma boa relação com os professores baseada na confiança proporciona aos jovens aprendizagens importantes para a aquisição de capacidades psicossociais e capacidades que ajudem à aquisição da autonomia, além da natural aquisição de conhecimentos, associada ao processo ensino-aprendizagem. Estas capacidades podem ajudar os adolescentes a gerirem melhor as suas relações, inclusive a relação com a mãe durante o processo de individuação. A relação aluno-professor pode favorecer o estímulo do aluno, nomeadamente no que concerne 
ao desenvolvimento de capacidades individuais e psicossociais (Mota \& Matos, 2014a), que ajudam a uma melhor gestão emocional e relacional, inclusive na relação com a mãe. Assim, o apoio social do professor percebido pelo adolescente e jovem é uma mais-valia, podendo mesmo vir a diminuir o impacto de acontecimentos negativos ao longo do seu desenvolvimento (DuBois et al., 2002), podendo desta forma ser um fator facilitador em caso de divórcio parental.

Para a negação do desejo de ligação à mãe e ao pai verifica-se que o sexo, a configuração familiar e a percepção do suporte social contribuem de forma significativa para a sua predição, sendo que em relação à mãe acresce a idade. Assim, verifica-se que a dimensão que mais contribui para a predição da negação do desejo de ligação ao pai e à mãe é mais uma vez a percepção do suporte da família, verificando-se que maiores níveis de percepção do suporte familiar predizem menores níveis de negação do desejo de ligação ao pai e à mãe. Jovens que sintam suporte, segurança e ao mesmo tempo autonomia para poderem explorar o mundo, não sentem necessidade de negar a proximidade afetiva e o suporte, nem de se defenderem e criar resistências, uma vez que os elementos da família ajudam a que seja mantido um equilíbrio entre o suporte $\mathrm{e}$ a liberdade. Fleming (2003) refere que é importante o jovem manter um equilíbrio entre a sua individuação e a ligação afetiva aos pais. Para Lidchi e Eisenstein (2004), o jovem desenvolve na sua família relações pautadas pela confiança, quando esta respeita equilibradamente a sua autonomia e a prestação de proteção e suporte.

Também o sexo contribui para a explicação dos modelos, sendo que os maiores níveis de negação do desejo de ligação ao pai e à mãe são atribuídos ao sexo masculino, o que poderá ser explicado pela diferença de sexos na envolvência afetiva com os outros. Os rapazes por reportarem uma postura mais externalizante, apresentam um maior desinvestimento nas suas relações afetivas do que as raparigas (Rocha, 2008). Um estudo de Allen e Stoltenberg (1995), com 182 jovens cuja média de idades era de 18,97 anos, verificou que enquanto o sexo feminino apresenta um ganho maior de suporte familiar, bem como maior satisfação na qualidade do mesmo, investindo mais nos afetos e vida emocional, o sexo masculino vê as suas famílias como menos coesivas, percecionando-as como menos prestadoras de suporte interpessoal. Esta percepção pode levar a um menor investimento na relação familiar por parte do sexo masculino.

A percepção do suporte social geral também mostra a sua contribuição para a explicação dos dois modelos na medida em que uma maior percepção do suporte social prediz maior negação do desejo de ligação ao pai e à mãe. Estes resultados devem-se ao fato de que uma percepção de suporte social geral pode ocasionar nos indivíduos mais inseguros uma aparente autonomia face às suas figuras primordiais de afeto que parece, na nossa amostra, aumentar a negação do desejo de ligação com a mãe. Ou seja, o maior investimento nas relações externas pode causar nos adolescentes e jovens adultos uma desvinculação ilusória.

Os resultados mostram ainda que a idade contribui de forma significativa para a explicação do modelo da negação do desejo de ligação à mãe, sendo que são os indivíduos mais jovens (13-18 anos) que apresentam níveis mais elevados de negação do desejo de ligação à mãe. Repare-se que é nesta idade da adolescência, entre os 13 e os 17 anos, que os jovens desenvolvem uma grande necessidade de se voltarem para o exterior, procurando conhecer e explorar o meio em redor, investindo mais nas relações com os pares e desinvestindo nas relações com os pais (Fernandes, 2007). Dá-se então uma falsa ideia de desvinculação que segundo Rakoff (1978) consiste na facilidade com que os indivíduos, neste caso os adolescentes, se percebem, erradamente, como totalmente independentes. Esta ilusão da desvinculação surge juntamente com o aumento da negação do desejo de vinculação aos pais, sendo que nesta altura os pares assumem um papel relevante na vida dos jovens por toda a similitude e partilha de vivências.

Finalmente, relativamente à negação do desejo de ligação ao pai, também a configuração familiar contribui de forma significativa para 0 modelo, sendo que são os jovens pertencentes a famílias divorciadas que apresentam níveis mais 
elevados de negação do desejo de ligação ao pai, o que pode ser explicado pelo grande número de jovens que após um divórcio parental vive com a figura materna, diminuindo assim as ligações afetivas à figura paterna (Brito, 2007). Esta situação torna-se particularmente relevante quando no seio da separação existem conflitos de lealdade que recriam nos jovens sentimentos de triangulação e coligação (Mota \& Matos, 2014b).

Relativamente ao modelo da individuação bem-sucedida ao pai e à mãe é possível observar que a configuração familiar e a percepção do suporte social contribuem para a sua predição, sendo que à individuação bem-sucedida à mãe acresce o sexo. Assim, a percepção do suporte da família prediz maiores níveis e de individuação bem-sucedida ao pai e à mãe. Este fato remete para a família enquanto promotora de uma individuação bem-sucedida e de um desenvolvimento de autonomia nos jovens e adolescentes. De fato, tal como os resultados têm evidenciado, um ambiente familiar pautado por relações seguras, às quais os jovens sabem que podem recorrer para a resolução de problemas ou para receberem apoio afetivo, de forma equilibrada com a autonomia dos jovens, torna-se propício a uma individuação bem-sucedida. Noom e colaboradores (1999) evidenciam no seu estudo que um melhor suporte afetivo dos pais facilita o desenvolvimento da formação da identidade e autonomização dos adolescentes. Também um estudo de Beyers e Goossens (2004) com 281 adolescentes com idades compreendidas entre os 18 e os 24 anos verificou que a relação dos filhos com os pais é um processo dinâmico, onde ocorre uma separação conjuntamente com mudanças nos modelos parentais. Assim, os pais evidenciam-se menos exigentes e controladores, mantendo a sua disponibilidade para dar suporte aos filhos, promovendo deste modo o desenvolvimento da autonomia dos jovens (Beyers \& Goossens, 2004). Um estudo de Mota e Matos (2013), com 538 jovens adultos portugueses entre os 18 e os 30 anos, concluiu que a percepção de cuidados recíprocos e a proximidade na relação parental modera os efeitos negativos dos conflitos interparentais no desenvolvimento do processo de individuação. O referido estudo evidencia, assim, a importância da qualidade da relação com os pais para a individuação bem-sucedida. As mesmas autoras referem que uma elevada percepção do cuidado parental diminui possíveis sentimentos de rejeição e retração que podem surgir em situações de conflitos interparentais, diminuindo assim possíveis efeitos negativos no desenvolvimento de uma individuação bem-sucedida. Também a configuração familiar explica significativamente os modelos, na medida em que são os jovens pertencentes a famílias intactas que predizem uma melhor qualidade na individuação ao pai e à mãe. Este resultado mostra que a qualidade da relação entre o adolescente e os pais é crucial para o processo de individuação, na medida em que favorece o desenvolvimento da autonomia e da identidade (Fleming, 2005).

\section{Considerações Finais}

A família é uma base de suporte muito importante para um saudável e equilibrado desenvolvimento dos adolescentes e mesmo dos jovens adultos. Alterações ocorridas neste núcleo de relações podem afetar de alguma forma esse desenvolvimento. O divórcio é uma dessas situações, sendo que a presente investigação mostrou que são os jovens pertencentes a famílias intactas apresentam maior percepção do suporte da família e níveis mais elevados de individuação bem-sucedida aos pais. Este fato poderá estar relacionado com os conflitos passíveis de ocorrer em situações de divórcio e a possíveis separações e quebras de vínculo com determinadas figuras da família. De um modo geral, todos os relacionamentos estabelecidos (família, pares, professores) e a percepção que se faz dos mesmos contribuem de forma significativa para o desenvolvimento dos adolescentes e jovens adultos. Assim, o suporte social e a percepção do mesmo mostraram predizer de forma significativa processos de individuação bem-sucedidos aos pais, na medida em que prevê o desenvolvimento de uma manutenção do laço aos pais de mais qualidade, uma menor negação do desejo de proximidade aos pais e, por conseguinte, individuações aos pais bem-sucedidas. 
Assim, futuras investigações poderão direcionar-se para estudos do tipo longitudinal, de modo a acompanhar o desenvolvimento dos jovens no âmbito da individuação. Seria também pertinente introduzir novas variáveis que possam explicar melhor o processo de individuação. Variáveis como a vinculação aos pais e o conflito interparental, são também pertinentes para a investigação, bem como abranger outro tipo de populações, como por exemplo, jovens já inseridos no mercado de trabalho, por poderem apresentar diferentes características em termos da individuação, autonomia e maturidade.

O presente estudo apresenta como limitações o reduzido número de jovens pertencentes a famílias divorciadas, em comparação de proporcionalidade com as famílias intactas, bem como a discrepância na amostra entre sexos, comportando um número superior do sexo feminino. Por fim, também o carácter de auto relato dos instrumentos de avaliação se apresenta como uma limitação, mostrando-se desta forma importante a inclusão de entrevista em futuras investigações.

\section{Referências}

Allen, S., \& Stoltenberg, C. (1995). Psychological separation of older adolescents and young adults from their parents: An investigation of gender differences. Journal of Counseling and Development, 73, 542-546.

Amato, P. (2000). Consequences of divorce for adults and children. Journal of Marriage and the Family, 62(4), 1269-1287. doi:10.1111/j.17413737.2000.01269.x

Amato, P., \& Afifi, T. (2006). Feeling caught between parents: Adult children's relations with parents and subjective well-being. Journal of Marriage and Family, 68, 222-235. doi:10.1111/j.17413737.2006.00243.x

Amato, P. R., \& Anthony, C. J. (2014). Estimating the effects of parental divorce and death with fixed effects models. Journal of Marriage and Family, 76(2), 370-386. doi:10.1111/jomf.12100

Amato, P., \& Cheadle, J. (2008). Parental divorce, marital conflict and children's behavior problems: A comparison of adopted and biological children. Social Forces, 86(3), 1139-1161. doi:10.1353/sof.0.0025
Amato, P., \& Dorius, C. (2010). Fathers, children, and divorce. In M. E. Lamb (Ed.), The role of the father in child development $\left(5^{\text {th }}\right.$ ed., pp. $177-$ 194). Toronto, Canada: Wiley.

Amato, P., \& Sobolewski, J. (2001). The effects of divorce and marital discord on adult children's psychological well-being. American Socialogical Review, 66, 900-921. doi:10.2307/3088878

Antunes, C. (2006). Evolução diferencial da auto-estima e do autoconceito académico na adolescência (Tese de doutorado, Faculdade de Psicologia e de Ciências da Educação, Universidade do Porto, Portugal).

Antunes, C., \& Fontaine, A. (1995). Diferenças na percepção de apoio social na adolescência: Adaptação de uma escala, o "Social Support Appraisals" (SSA) de Vaux et al. (1980). Cadernos de Consulta Psicológica, 10-11, 115127.

Antunes, C., \& Fontaine, A. (2005). Percepção de apoio social na adolescência: Análise fatorial confirmatória da escala social support appraisals. Paidéia (Ribeirão Preto), 15(32), 355-366. doi:10.1590/S0103-863X2005000300005

Arnett, J. J. (2006). Emerging adulthood in Europe: A response to Bynner. Journal of Youth Studies, 9, 111-123. doi:10.1080/13676260500523671

Arnett, J. J. (2007). Emerging adulthood: What is it, and what is it good for? Child Development Perspectives, 1(2), 68-73. doi:10.1111/j.17508606.2007.00016.x

Beato, A. (2008). "Adolescencer” entre relações: Parentalidade, amizade e amorosidade: que contributos na transição para a idade adulta? (Dissertação de mestrado, Faculdade de Psicologia e de Ciências da Educação, Universidade de Lisboa, Portugal).

Beyers, W., \& Goossens, L. (2004). Contributions of parenting to the separation-individuation process in late adolescence: A three-wave longitudinal study. In G. Masche (Chair), The development of parenting behaviors and of parent-child relationships in adolescence: Origins of autonomy. Symposium conducted at the $10^{\text {th }}$ biennial meeting of the Society for Research on Adolescence (SRA), Baltimore, MD.

Blos, P. (1967). The second individuation process of adolescence. The Psychoanalytic Study of the Child, 22, 162-186. 
Brito, L. (2007). Família pós-divórcio: A visão dos filhos. Psicologia Ciência e Profissão, 27(1), 32-45. doi:10.1590/S1414-98932007000100004

Brown, C. (2009). Ameliorating the effects of divorce on children. Journal of the American Academy of Matrimonial Lawyers, 22, 461-482.

Bynner, J. (2005). Rethinking the youth phase of the life-course: The case for emerging adulthood? Journal of Youth Studies, 8, 367-384. doi:10.1080/13676260500431628

Coob, S. (1976). Social support as a moderator of life stress. Psychosomatic Medicine, 38, 300-314.

DuBois, D. L., Burk-Braxton, C., Swenson, L. P., Tevendale, H. D., Lockerd, E. M., \& Moran, B. L. (2002). Getting by with a little help from self and others: Self-esteem and social support as resources during early adolescence. Developmental Psychology, 38(5), 822-839. doi:10.1037/00121649.38.5.822

Fernandes, H. (2007). O bem-estar psicológico em adolescentes: Uma abordagem centrada no florescimento humano (Tese de doutorado, Universidade de Trás-os-Montes e Alto Douro, Vila Real, Portugal).

Fleming, M. (2003). O risco de não correr risco nenhum: Impasses do desenvolvimento psicológico adolescente. Revista Portuguesa de Psicanálise, 24, 97-105.

Fleming, M. (2005). Entre o medo e o desejo de crescer: Psicologia da adolescência. Porto, Portugal: Afrontamento.

Freeman, H. S., \& Newland, L. A. (2002). Family transitions during the adolescent transition: Implications for parenting. Adolescence, 37, $457-$ 475 .

Furman, W. (2001). Working models of friendships. Journal of Social and Personal Relationships, 18, 583-602. doi:10.1177/0265407501185002

Hack, S., \& Ramires, V. (2010). Adolescência e divórcio parental: Continuidades e rupturas dos relacionamentos. Psicologia Clínica, 22(1), 8597. doi:10.1590/S0103-56652010000100006

Heller, K., Swindle, K., \& Dunsenbury, L. (1986). Component social support processes: Comments and integration. Journal of Consulting and Clinical Psychology, 54, 466-470.

Hombrados-Mendieta, M. I., Gomez-Jacinto, L., Dominguez-Fuentes, J. M., Garcia-Leiva, P., \& Castro-Travé, M. (2012). Types of social support provided by parents, teachers, and classmates during adolescence. Journal of Community Psychology, 40(6), 654-664. doi:10.1002/ jcop. 20523

Ikiz, F., \& Cakar, F. (2010). Perceived social support and self-esteem in adolescence. Procedia Social and Behavioral Sciences 5, 2338-2342. doi:10.1016/j.sbspro.2010.07.460

Kruse, J., \& Walper, S. (2008). Types of individuation in relation to parents: Predictors and outcomes. International Journal of Behavioral Development, 32(5), 390-400. doi:10.1177/0165025408093657

Leme, V., Del Prette, Z., \& Coimbra, S. (2015). Social skills, social support and well-being in adolescents of different family configurations. Paidéia (Ribeirão Preto), 25(60), 9-17. doi:10.1590/1982-43272560201503

Lidchi, V., \& Eisenstein, E. (2004). Adolescentes e famílias no contexto médico. In J. Mello Filho (Ed.), Doença e família (pp. 217-231). São Paulo, SP: Casa do Psicólogo.

Mahler, M., Pine, F., \& Bergman, A. (1975). The psychological birth of the human infant: Symbiosis and individuation. London: Hutchinson.

Matos, P. M., \& Hüsgen, T. (2009). Munich Individuation Test of Adolescence (MITA). Adaptação para a população portuguesa. Manuscrito não publicado, Faculdade de Psicologia e de Ciências da Educação, Universidade do Porto.

Meeus, W., Iedema, J., Maassen, J., \& Engels, R. (2005). Separation-individuation revisited: On the interplay of parent-adolescent relations, identity and emotional adjustment in adolescence. Journal of Adolescence 28, 89-106. doi:10.1016/j.adolescence.2004.07.003

Mota, C. P., \& Matos, P. M. (2011). Adolescência e conflitos parentais: Uma perspetiva de resiliência. In P. Matos \& M. Costa (Eds.), Temas contemporâneos de intervenção psicológica com famílias. Porto, Portugal: LivPsic.

Mota, C. P., \& Matos, P. M. (2013). Conflitos interparentais e processos de individuação em adultos emergentes portugueses: O papel dos conflitos de lealdade. Psicologia: Teoria $e$ Pesquisa, 29(3), 267-276. doi:10.1590/S010237722013000300004

Mota, C. P., \& Matos, P. M. (2014a). Padres, profesores y pares: contribuciones para la autoestima y coping en los adolescentes. Anales de 
Psicologia, 30(2), 656-666. doi:10.6018/analesps.30.2.161521

Mota, C. P., \& Matos, P. M. (2014b). Relación parental, autoestima y depression en jóvenes portugueses: implicaciones de los conflictos interparentales, coligación y triangulación. Universitas Psychologica, 13(3), 907-922.

Moura, O., \& Matos, P. M. (2008). Vinculação aos pais, divórcio e conflito inter-parental em adolescentes. Psicologia, 22(1), 127-152. doi:10.17575/rpsicol.v22i1.34

Noom, M., Dekovic, M., \& Meeus, W. (1999). Autonomy, attachment and psychosocial adjustment during adolescence: A double-edged sword? Journal of Adolescence, 22, 771-783. doi:10.1006/jado.1999.0269

Parmiani, L., Iafrate, R., \& Giuliani, C. (2012). Loyalty conflict, feelings of unfairness, and young adults' individuation difficulties in separated and nonseparated families. Journal of $D i$ vorce \& Remarriage, 53(5), 386-401. doi:10.10 $80 / 10502556.2012 .682889$

Pinheiro, M., \& Ferreira, J. (2005). A perceção do suporte social da família e dos amigos como elementos facilitadores da transição para o ensino superior. In Atas do VIII congresso galaico-português de psicopedagogia (pp. 467-485). Braga, Portugal: Centro de Investigação em Educação, Universidade do Minho.

Rakoff, V. (1978). The illusion of detachment. Adolescent Psychiatry, 6, 119-129.

Ramalho, C. (2008). (Os) nós e os laços: Vinculação, suporte social e bem-estar em jovens adultos (Dissertação de mestrado, Faculdade de Psicologia e de Ciências da Educação, Universidade de Lisboa, Portugal).

Ramires, V. (2004). As transições familiares: A perspectiva de crianças e pré-adolescentes. Psicologia em Estudo, 9(2), 183-193. doi:10.1590/ S1413-73722004000200005
Rocha, M. (2008). O desenvolvimento das relações de vinculação na adolescência: Associações entre contextos relacionais com pais, pares $e$ par amoroso (Tese de doutorado, Faculdade de Psicologia e de Ciências da Educação, Universidade do Porto, Portugal).

Sérgio, M. B. B. (2006). A percepção do suporte social dos adolescentes com ou sem deficiência motora. Enfermeria Global, 8, 1-10.

Souza, R. M., \& Ramires, V. R. (2006) Amor, casamento, família, divórcio... e depois, segundo as crianças. São Paulo, SP: Summus.

Storksen, I., Roysamb, E., Moum, T., \& Tambs, K. (2005). Adolescents with a childhood experience of parental divorce: A longitudinal study of mental health and adjustment. Journal of Adolescence, 28, 725-739. doi:10.1016/j.adolescence. 2005.01 .001

Walper, S., Kruse, J., Noack, P., \& Schwarz, B. (2009). Parental separation and adolescents' felt insecurity with mothers: Effects of financial hardship, interparental conflict, and maternal parenting in east and West Germany. Marriage \& Family Review, 36(3), 115-145. doi:10.1300/ J002v36n03_07

Walper, S., Schwarz, B., \& Jurasic, S. (1996). Entwicklung und Erprobung des Münchner Individuationstests. Berichte aus der Arbeitsgruppe "Familienentwicklung nach der Trennung". München, Deutschland: Ludwig-Maximilians UniversitätMünchen.
Recebido: 18/02/2015

$1^{a}$ revisão: $17 / 10 / 2015$

$2^{a}$ revisão: $04 / 11 / 2015$ Aceite final: 12/11/2015 